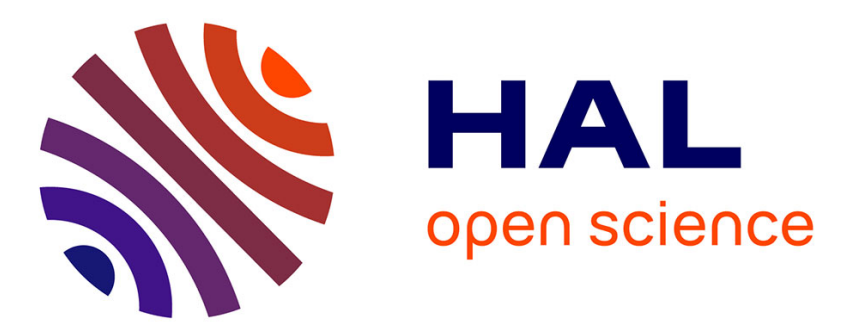

\title{
Charges injection investigation at metal/dielectric interfaces by Kelvin Probe Force Microscopy
}

Florian Mortreuil, Christina Villeneuve-Faure, Laurent Boudou, Kremena

Makasheva, G. Teyssedre

\section{- To cite this version:}

Florian Mortreuil, Christina Villeneuve-Faure, Laurent Boudou, Kremena Makasheva, G. Teyssedre. Charges injection investigation at metal/dielectric interfaces by Kelvin Probe Force Microscopy. 2016 IEEE International Conference on Dielectrics (ICD), Jul 2016, Montpellier, France. pp.493-496, 10.1109/ICD.2016.7547650 . hal-02324471

\section{HAL Id: hal-02324471 \\ https://hal.science/hal-02324471}

Submitted on 1 Nov 2019

HAL is a multi-disciplinary open access archive for the deposit and dissemination of scientific research documents, whether they are published or not. The documents may come from teaching and research institutions in France or abroad, or from public or private research centers.
L'archive ouverte pluridisciplinaire HAL, est destinée au dépôt et à la diffusion de documents scientifiques de niveau recherche, publiés ou non, émanant des établissements d'enseignement et de recherche français ou étrangers, des laboratoires publics ou privés. 


\title{
Charges injection investigation at metal/dielectric interfaces by Kelvin Probe Force Microscopy
}

\author{
F. Mortreuil, C. Villeneuve-Faure, L. Boudou, K. Makasheva, G. Teyssedre
}

LAPLACE (Laboratoire Plasma et Conversion d'Energie), Université de Toulouse, CNRS, UPS, INPT ; 118 route de Narbonne, F-31062 Toulouse cedex 9, France.

\begin{abstract}
Charges injection at metal/dielectric interface and their motion in silicon nitride layer is investigated using samples with embedded lateral electrodes and surface potential measurement by Kelvin Probe Force Microscopy (KPFM). Bipolar charge injection was evidenced using this method. From surface potential profile, charge density distribution is extracted by using Poisson's equation. The evolution of the charge density profile with polarization bias and depolarization time was also investigated.
\end{abstract}

Keywords-charge injection, Kelvin Probe Force Microscopy, charges density profile, thin dielectric

\section{INTRODUCTION}

Charges accumulation in dielectrics has been extensively studied during the last decades. The approach is based on charge probing methods providing information on charge localization at micrometer scale -space charge measurement [1]- or more integral scale (thermo-stimulated current [2] or surface potential measurements for example). The information gained cannot be directly connected to microstructure of the material because of the rather coarse resolution of the methods. Therefore the information is treated as representative of bulk homogeneous material physics. The approach is no longer sustainable in the modern times owing to the development of nanoscale components (microelectronic and microsystems) or the availability of new materials (nanocomposite for example). Indeed, review about dielectric breakdown in MOS structure highlights that injected and trapped charges exert a strong impact on components reliability [3]. Whereas main physical phenomena occur at nanometer-scale, relevant parameters, as energy barrier to injection or interface/bulk defect energetic features are extracted from macroscale measurements [4]. As a consequence of the limited information, simplified schemes are produced, leading to a failure of theoretical predictions to explain different measurements. For example, the origin of anode hole injection and heterogeneous breakdown in metal$\mathrm{SiO}_{2}$-metal structure is still a controversy subject [5].

Whatever the considered application field, it is actually necessary to implement diagnostic means which provide information at relevant scale, that is to say at the scale level where the physical processes occur. Along this scheme, for investing electronic carriers generation, or transport in nanocomposite structures, it is required to probe and control processes acting at interfaces (outer as metal-dielectric interface, or internal as in nanocomposites).
To reach this goal, incremental improvement of currently available perturbation-based charge probing methods appears problematic. Besides, electrical modes derived from Atomic Force Microscopy (AFM) were extensively used during the past decades. According to bibliographical review three main experimental techniques can be used to investigate charges in dielectrics, either dynamically or statically:

(i) Current measurement using Conductive-AFM permits to investigate locally conduction process from leakage current to breakdown [6]. But issues like surface collection indetermination limit the extraction of physical parameters as energy barrier to injection and the measurement requires hypotheses on the bulk transport [7].

(ii) Electrostatic Force Microscopy (EFM) [8] measurements provide estimates of surface charge density in very thin dielectric materials (for sample thickness less than $5 \mathrm{~nm}$ ) and is expected to be sensitive to single charge detection [9]. But its sensitivity to image charges and to applied bias now limits its use.

(iii) Kelvin Probe Force Microscopy (KPFM) [10], through its ability to probe directly the potential induced by electrostatic charges stored in dielectric materials, is preferred to EFM to probe charges. Whereas KPFM reaches atomic resolution and single charge probing under vacuum and in Non-Contact mode on isolated molecules adsorbed on well-adapted surface [11], the spatial resolution is much more coarse (25-to-100nm) in the conditions of nanoscale characterization of trapped charges (air environment, tapping mode KPFM, unknown charge distribution in volume, etc.).

A convenient way to investigate charge storage using AFM consists in generating charges in the dielectric using a biased AFM tip as depicted in Fig. 1.a. The potential profile (as in KPFM) and its time dependence should provide information on the charge quantity, localization and motion. However, due to experimental artefact (apparent height, convolution of potential distribution due to the long range of electrostatic forces, etc.) quantitative charge density estimation is not straightforward. In the configuration of Fig. 1.a, the main issue concerns the vertical distribution of charges which should be known to manage to determine volume charges density [12]. However, the KPFM does not provide information on that. There are some available models for KPFM, but they are based on strong hypotheses about the charge localization profile in volume of the studied dielectric [12-13]. One way for overcoming this 
drawback is to use lateral electrodes to inject charges (Fig. 1.b). Supposing that the charges are injected uniformly in volume along each electrode, charges injection study which is a 3D problem in Fig. 1.a. becomes a 2D problem in Fig. 1.b. The use of lateral electrodes is an interesting way to investigate charges injection phenomena. This configuration was already used to study charges transport in organic semiconductor using EFM [14-15] or KPFM [16]. Interesting results were obtained, mainly combining with current measurement $[15,17]$, but a strong dependence on the film morphology is observed [14], which makes interpretation tricky.

The aim of this contribution is to investigate the feasibility of measuring surface potential modification due to charges injected by lateral electrodes in case of inorganic insulating materials. In the first part of the contribution, the experimental set-up will be described. In the following surface potential modification during polarization and depolarization step will be investigated by KPFM. Finally, a first attempt to extract charge density profiles from surface potential measurements is proposed.
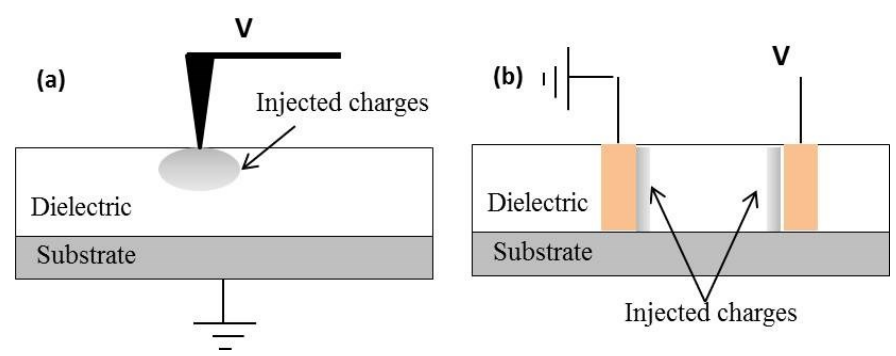

Fig. 1. Hypothesized charge injection patterns using (a) biased conductive AFM-tip and (b) biased lateral electrodes.

\section{EXPERIMENTS}

In this study, a $\mathrm{SiN}_{\mathrm{x}}$ dielectric layer processed in High Frequency Plasma Enhanced Chemical Vapor Deposition process [18] was used as thin dielectric layer deposited on $\mathrm{Si}$ substrate. Using lift-off process, 70nm-thick aluminum electrodes were embedded in the $270 \mathrm{~nm}$-thick $\mathrm{SiN}_{\mathrm{x}}$. This geometry ensures an intimate metal/dielectric contact (verified using Scanning Electron Microscopy on cross-section) and small surface roughness (less than $5 \mathrm{~nm}$ height difference between the $\mathrm{SiN}_{\mathrm{x}}$ and the Al-electrodes). Finally, a $5 \mathrm{~nm}$ passivation cover-layer of $\mathrm{SiN}_{\mathrm{x}}$ was deposited over the electrode. The upper dielectric surface ensures isolation of the AFM tip from the electrodes when biasing the electrodes. In addition, the resulting surface is flat enough to reduce topography artefacts on the surface potential profile. Fig. 2 represents the final structure observed by optical microscopy. Structures with different inter electrodes distances were designed (from $5 \mu \mathrm{m}$ to $40 \mu \mathrm{m}$ ).

The AM-KPFM (Amplitude Modulation KPFM) surface potential measurements were realized on a Bruker Multimode 8 set-up using Pt-coated silicon tip (SCM-PIT provided by Bruker) in lift mode (10nm lift distance). All measurements were performed under dry nitrogen atmosphere after sample annealing for $4 \mathrm{~min}$ on $120^{\circ} \mathrm{C}$ hot plate to remove the naturally adsorbed on the surface water layer. Charges were injected applying symmetric bias $(\Delta \mathrm{V}=10 \mathrm{~V}$ to $20 \mathrm{~V})$ on the lateral electrodes (Fig. 2). The electric field was applied during fixed time ( 1 to $3 \mathrm{~h}$ ). The measurements were realized about 60 s after removing the bias voltage. The electrodes were normally shortcircuit.

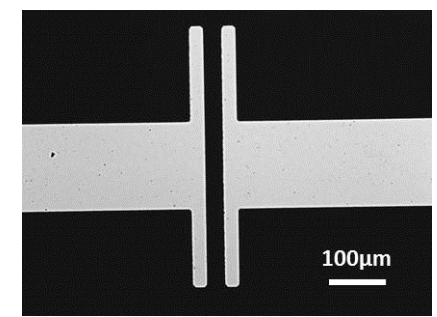

Fig. 2. Optical microscopy picture (top view) Al-lateral electrodes embedded in $\mathrm{SiN}_{\mathrm{x}}$. Inter-electrode distance of $20 \mu \mathrm{m}$-width.

\section{RESULTS AND INTERPRETATION}

\section{A. Polarization step}

Fig.3.a. represents a topography image realized over the electrodes and the dielectric layer. The distance between electrodes is $10 \mu \mathrm{m}$ and the maximum height difference between electrode and $\mathrm{SiN}_{\mathrm{x}}$ is $4.8 \mathrm{~nm}$. Before polarization, the surface potential profile between the electrodes is flat. After the polarization ( $1 \mathrm{~h}$ at $\Delta \mathrm{V}=20 \mathrm{~V}$ the potential profile is no longer homogeneous as depicted in (Fig. 3.b.). The all potential variation is found over the dielectric between the electrodes. These potential variations are not related to topographical perturbation since the metal/dielectric interface is smooth. Negative charges are injected close to cathode and positive charges close to anode. The potential profile appears fairly homogeneous when moving in a direction parallel to the electrode.



Fig. 3. (a) Topography and (b) surface potential cartography after $1 \mathrm{~h}$ polarization at $20 \mathrm{~V}$ applied on lateral electrodes separated by $10 \mu \mathrm{m}$. Cathode to the left $(-10 \mathrm{~V}$ applied) and anode to the right $(+10 \mathrm{~V}$ applied on right electrode).

Fig. 4 represents potential profiles induced by injected charges for different applied potentials. The interfaces between layers have been defined following AFM topographic images. Note that the offset appearing in some cases over the electrodes could be due either to charge build-up in the dielectric passivation layer, or to uncontrolled electrodes short-circuiting. Results highlight that when the applied bias (i.e. electric field) increases, the maximum potential increases whereas the position of the peak moves slightly towards the bulk of the material. The peak full width at half-maximum (FWHM) is about $2 \mu \mathrm{m}$ in all cases. Positive and negative peaks have 
roughly the same magnitude (though apparently a bit larger for positive charges), meaning that electrons and holes appear injected with the same efficiency.

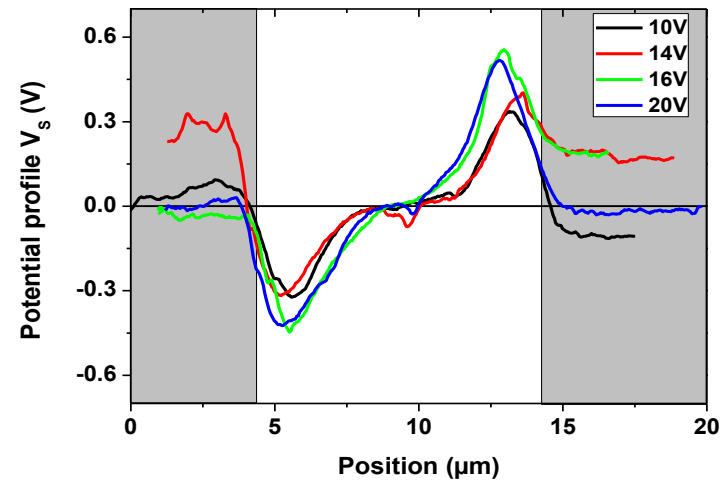

Fig. 4. Surface potential profile $\mathrm{V}_{\mathrm{S}}(\mathrm{x})$ between electrodes separated by $10 \mu \mathrm{m}$ measured after $180 \mathrm{~min}$ of polarization at different applied voltages.

\section{B. Extraction of charge density}

The simplest hypothesis for determining charge quantity consists in supposing that the measured potential corresponds actually to the inter-electrode potential distribution. This appears as reasonable hypothesis given the fact that the lift distance and the tip apex are small in respect to the interelectrode distance. More sophisticated EFM modelling has confirmed the validity of the approximation. From the surface potential $\mathrm{V}_{\mathrm{S}}(\mathrm{x})$, charges density $\rho(\mathrm{x})$ has been extracted by using the Poisson's equation:

$$
\rho(\mathrm{x})=\varepsilon_{0} \varepsilon_{r} \frac{d^{2} V_{S}}{d x^{2}},
$$

with $\varepsilon_{0}$ is the vacuum permittivity, $\varepsilon_{\mathrm{r}}$ is the $\mathrm{SiN}_{\mathrm{x}}$ relative permittivity $\left(\varepsilon_{\mathrm{r}}=5.7\right)$ and $\mathrm{x}$ the lateral position.

Applying this treatment (equation 1) to surface potential profiles depicted on Fig. 4, charge density profiles were extracted as represented in Fig. 5. It is confirmed that negative space charge is formed adjacent to the cathode and positive charges to the anode. Moreover, image charges are formed on the electrodes. To provide estimates of profile to profile variation of derived charge density, the potential profile over large area as in Fig. 3 exhibits a $8 \%$ variation of maximum potential. This leads to a $10 \%$ variation on charges density. Even if the noise level (not due to profile-to-profile dispersion) is important and would require signal treatment improvement, some information could be extracted from the results. First, the charge density profiles have roughly the same shape; the charged region extends up to about $2 \mu \mathrm{m}$ from the electrode/dielectric interface for both electrons and holes. Moreover the maximum density increases with polarization bias; for example, for holes the maximum density is equal to $25 \mathrm{C} / \mathrm{m}^{3}$ and $48 \mathrm{C} / \mathrm{m}^{3}$ for polarization at $10 \mathrm{~V}$ and $20 \mathrm{~V}$, respectively. At this point and with the associated noise, no difference between positive and negative charge build up can be put forward.

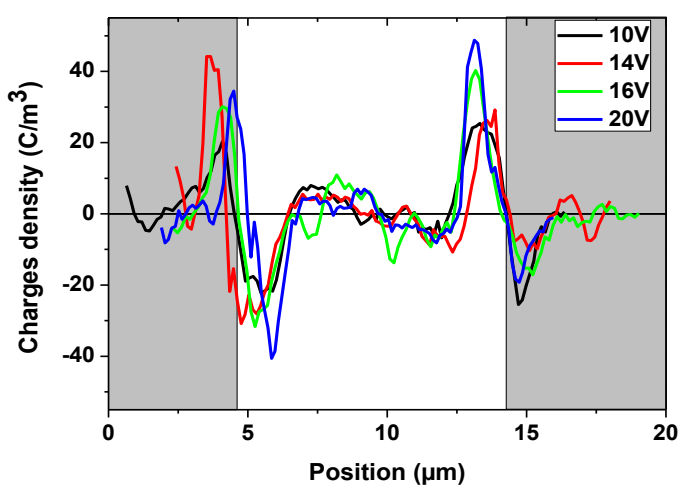

Fig. 5. Charge density profiles extracted from Fig. 4 for different polarization conditions (inter-electrode distance of $10 \mu \mathrm{m}$ ) Charges release during depolarization steps

Fig.6 represents the evolution of surface potential profile with time after polarization under $20 \mathrm{~V}$. With the time increasing, the surface potential maximum decreases; its position moves away from the interface and the potential profile broadens. Potential profiles of Fig.6 were treated using equation 1, and the extracted parameters relevant to the charge density profiles are given in Table 1. With time after charging, the charge density decreases and the peak moves away from the electrode/dielectric interface. In the same time charge density distribution broadens (the FWHM changes from $1.5 \mu \mathrm{m}$ to $2 \mu \mathrm{m}$ for initial and after $247 \mathrm{~min}$, respectively. For these results, the positive charges density appears larger than the negative ones, a feature somewhat clearer than in the previous results presented in Fig. 5.

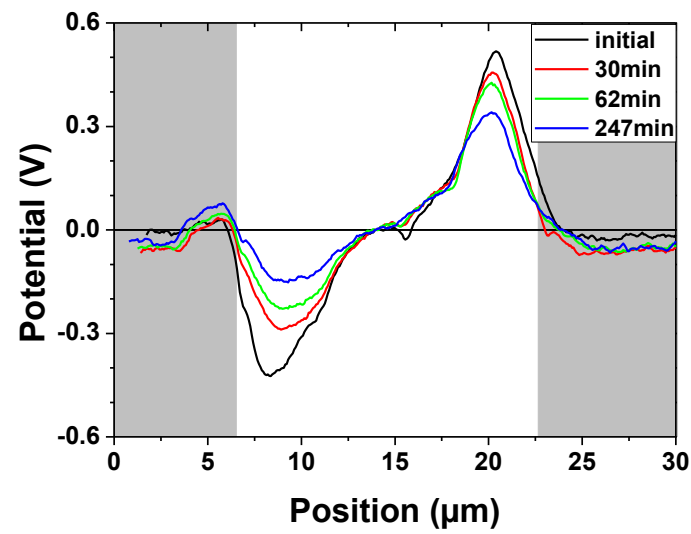

Fig. 6. Evolution of surface potential profile with time after $3 \mathrm{~h}$ at $20 \mathrm{~V}$ (inter-electrode distance of $15 \mu \mathrm{m}$ ).

\begin{tabular}{|l|l|l|l|l|l|}
\hline & & initial & $30 \mathrm{~min}$ & $62 \mathrm{~min}$ & $247 \mathrm{~min}$ \\
\hline \multirow{2}{*}{$\begin{array}{l}\text { Maximum } \\
\left(\mathrm{C} / \mathrm{m}^{3}\right)\end{array}$} & Holes & 16 & 14 & 13 & 9 \\
\cline { 2 - 6 } & Electrons & 12 & 6 & 4 & 4 \\
\hline \multirow{2}{*}{$\begin{array}{l}\text { Position } \\
(\mu \mathrm{m})\end{array}$} & Holes & 1 & 1.5 & 1.5 & 1.5 \\
\cline { 2 - 6 } & Electrons & 1.5 & 2 & 2 & 2 \\
\hline
\end{tabular}

Table 1. Evolution of charge density maximum and position related to electrode/dielectric interface with time after polarization for $3 \mathrm{~h}$ under $20 \mathrm{~V}$. 


\section{Discussion}

The above presented results show the feasibility of measuring charge build-up in thin dielectric layers using embedded electrodes. Charge injection from Al electrodes into $\mathrm{SiN}_{\mathrm{x}}$ is clearly revealed. In the current measurement conditions, the apparent penetration depth of the charges ranges from 1 to 2 $\mu \mathrm{m}$, with polarization time of several hours, but with moderate fields $(1-2 \mathrm{kV} / \mathrm{mm})$. Charge densities of the order of $20 \mathrm{C} / \mathrm{m}^{3}$ were measured producing residual space charge induced-field of the order of $0.5 \mathrm{kV} / \mathrm{mm}$, i.e. a substantial fraction from the previously applied field. The width of the potential profiles reported herein is consistent with the one obtained $(1-2 \mu \mathrm{m})$ using more conventional KPFM measurements following charging of similar materials $\mathrm{SiN}_{\mathrm{x}}$ with an AFM tip [18]. Hence, at this stage, though the real spatial resolution of the method is difficult to estimate, it can be anticipated that the potential shape is controlled by intrinsic material behavior rather than by instrument limitations. In another respect, it would be tempting to probe charges generated with applying higher fields, which could be reached by increasing bias voltage and/or decreasing inter-electrode distance. Three limitations can be anticipated along that goal, related to instrument security (voltage), resolution of the method/actual charge position (thickness) and flashover phenomenon appearance between the electrodes (both). The use of model materials with more resistance to charging could be a way to address the instrumental resolution limits.

\section{CONCLUSION}

In this paper the feasibility of using surface potential measurement by KPFM on lateral embedded electrodes to study charges injection was demonstrated. For different polarization conditions, charge density profiles were extracted by using Poisson's equation. Injected charges are located close to electrode/dielectric interface. The next step is now to determine a signal treatment in order to reduce noise on charges density profile and to determine the limit of the numerical method to extract charge profiles mainly for thin dielectric films.

\section{ACKNOWLEDGMENT}

This work was partly supported by the French RENATECH network.

\section{REFERENCES}

[1] N.H. Ahmed and N.N. Srinivas "Review of space charge measurements in dielectrics" IEEE Trans. Dielectr. Electr. Insul., vol. 4, pp. 644-656, 1997.

[2] Current meas $\rightarrow$ Laurent

[3] A. Ghetti, Gate Oxide Reliability: Physical and Computational Models. New York: Springer, 2004, pp. 201-258

[4] A. Martin, P. O'Sullivan, and A. Mathewson "Dielectric reliability measurement methods: a review", Microelectron. Reliab., vol. 38, pp. 37-72, 1998.

[5] J.F. Verweij and J.H. Klootwijk, "Dielectric breakdown I: A review of oxide breakdown", Microelectronics J., vol. 27, pp. 611-622, 1996.

[6] R. Foissac, S. Blonkowski, M. Kogelschatz, and P. Delcroix, "A relationship between statistical time to breakdown distribution and prebreakdown negative differentiel resistance at nanometric scale", J. Appl. Phys., vol. 116, p. 024505, 2014.

[7] O. Reid, K. Munechika, and D.S. Ginger, "Space charge limited current measurements on conjugated polymer film using conductive Atomic Force Microscopy", Nano Lett., vol. 8, pp. 1602-1609, 2008.

[8] G.C. Stevens and P.J. Baird, "Nano and Meso measurement methods in the study of dielectrics", IEEE Trans. Dielectr Electr. Insul., vol. 12, pp. 979-992, 2005.

[9] C. Schönenberger and S. Alvarado, "Observation of signle charge carriers by force microscopy", Phys. Rev. Lett., vol. 65, pp.3162-3164, 1990.

[10] M. Ishii, "Static states and dynamic behaviour of charges: observation and control by scanning probe microscopy", J. Phys.: Cond. Matter, vol. 22, p. 173001, 2010.

[11] L. Nony, A.S. Foster, F. Bocquet, and C. Loppacher, "Understanding the atomic-scale contrast in Kelvin Probe Force Microscopy" Phys. Rev. Lett., vol. 103, p.036802, 2009.

[12] . L. Borowik, K. Kusiaku, D. Theron, and T. Melin, "Calculating Kelvin force microscopy signals from static force field, Appl. Phys. Lett., vol. 96, p.103119, 2010.

[13] D.S.H. Charrier, M. Kemerik, B.E. Smalbrugge, T. Vries, and R.A.J. Janssen, "Real versus measured surface potentials in scanning Kelvin probe microscopy" ACS Nano, vol. 2, pp. 622-626, 2008

[14] M. Jaquith, E.M. Muller, and J.A. Marohn, "Time-resolved electrostatic force microscopy of charge trapping in polycristalline pentacene", J. Phys. Chem. B, vol. 111, pp.7711-7714, 2007.

[15] T.N. Ng, W.R. Silveira, and J.A. Marohn, "Dependence of charge injection on temperature, electric field and energetic disorder in an organic semiconductor", Phys. Rev. Lett., vol. 98, p. 066101, 2007.

[16] D.M. Taylor, D. Morris, and J.A. Cambridge, "Time evolution of the electric field at electrode interfaces with conducting polymers", Appl. Phys. Lett., vol. 85, pp. 5266-5268, 2004.

[17] W.R. Silveira and A. Marohn, "Microscopic view of charge injection in an organic semiconductor", Phys. Rev. Lett., vol. 93, p.116104, 2004.

[18] U. Zaghloul, G.J. Papaioannou, F. Coccetti, P. Pons, and R. Plana "A systematic reliability investigation of the dielectric charging process in electrostatically actuated MEMS based on Kelvin probe force microscopy", J. Micromech. Microeng., vol. 20, p. 064016, 2010. 